\title{
The prevention and treatment of peritoneal carcinomatosis from gastric cancer: A 2013 update
}

\author{
Ansaloni $\mathrm{L}^{1, *}$, Lotti $\mathrm{M}^{1}$, Campanati $\mathrm{L}^{1}$, Bertoli $\mathrm{P}^{1}$, Poiasina $\mathrm{E}^{1}$, Mandalà $\mathrm{M}^{2}$, Coccolini $\mathrm{F}^{1}$ \\ ${ }^{1}$ Unit of General Surgery I, Papa Giovanni XXIII Hospital, Bergamo, Italy \\ ${ }^{2}$ Unit of Medical Oncology, Papa Giovanni XXIII Hospital, Bergamo, Italy
}

\begin{abstract}
Gastric cancer is the fourth most common cancer and the second leading cause of cancer death in the world. Penetration of the gastric serosa and lymphatic spread are the two most important factors affecting prognosis in gastric cancer. Patients with peritoneal carcinomatosis from gastric cancer show a particularly poor prognosis. A few treatment strategies have been tested and proposed to increase survival rates. The cornerstone of treatment remains complete cytoreductive surgery associated with a different combination of chemotherapy regimen. It has been demonstrated as systemic adjuvant, systemic neoadjuvant and above all hyperthermic intraperitoneal chemotherapy improve the survival, when used in the prevention and treatment of peritoneal carcinomatosis from gastric cancer. Few new molecules have been introduced enhancing the effect of chemotherapy by biologically targeting its objective. However further studies are needed.
\end{abstract}

Keywords: gastric cancer; peritoneal metastasis; carcinomatosis; HIPEC; chemotherapy; neoadjuvant; adjuvant; treatment; prevention

\section{Introduction}

Gastric cancer (GC) is the fourth most frequent neoplasm with about one million new cases estimated to occur every year and the second most important cause of cancer death $(738,000$ estimated deaths yearly) worldwide [1, 2]. Metastatic dissemination in GC may occur through the hematic torrent or through the inside surface of the peritoneal cavity: this last condition is called peritoneal carcinomatosis (PC) and it's considered a stage IV of GC. Recent studies confirmed that PC is more frequent than metastases through hematic torrent, in fact only $40 \%$ of patients who died for GC have hepatic metastases, while $53-60 \%$ showing disease progression died with PC. So penetration of the gastric serosa and lymphatic spread are the two most important factors affecting prognosis in GC [3-5].PC becomes practically unavoidable when the gastric serosa is infiltrated by tumor [6]. Subsequently, up to half of the patients with advanced gastric cancer (AGC) will develop a peritoneal carcinomatosis from gastric cancer (PCGC) in spite of even radical surgery [7-10]. For these reasons PCGC is a quite common event in this neoplasm and is already present in $5-20 \%$ of patients explored for potentially curative resection $[6,11]$. Although there are several methods for detecting the presence of free peritoneal tumor cells (FPTCs) with different degrees of sensitivity [12-14], it should always been considered that
FPTCs in the washing could be identified in up to $24 \%$ of stage IB GC patients and up to $40 \%$ of those with stage II or III [15]. These findings explain why GC is considered a neoplasm with easy intraperitoneal spread, reflecting the fact that recurrence patterns after complete resection can vary, in different countries and different period times, from 10.2 to $33.9 \%$ as peritoneal recurrence alone and from 29.5 to $43.9 \%$ as peritoneal recurrence combined with other sites [16-20] (Table 1). The well-known mechanism of peritoneal spread of neoplastic cells of GC [21] explicates probably also the fact that peritoneal recurrence, although in a small percentage (1-2\%), can be observed even after potentially curative resection of early GC [22].

*Corresponding author: Ansaloni L, Unit of General Surgery I, Papa Giovanni XXIII Hospital, Piazza OMS 1, 24127 Bergamo, Tel.: +39 035 269310; Fax: +39 035 266898; Mobile: +39 328 9128533; E-mail: lansaloni@ospedaliriuniti.bergamo.it

Received 10 November 2012 Revised 3 January 2013 Accepted 10 January 2013 Published 17 January 2013

Citation: Ansaloni L, Lotti M, Campanati L, Bertoli P, Poiasina E, Mandalà M, Coccolini F (2013) The prevention and treatment of peritoneal carcinomatosis from gastric cancer: A 2013 update. J Cancer Res Ther 1: 54-59. doi:10.14312/2052-4994.2013-8

Copyright: (C) 2013 Ansaloni L et al. This is an open-access article distributed under the terms of the Creative Commons Attribution License, which permits unrestricted use, distribution and reproduction in any medium, provided the original author and source are credited. 
With the aim of updating knowledge in relation to the prevention and treatment of PCGC a review of the most recent literature was performed.

Table 1 Percentage of peritoneal recurrence alone and peritoneal recurrence combined with other sites among different recurrence patterns after complete resection of gastric cancer

\begin{tabular}{lll}
\hline \multicolumn{1}{c}{ Country, period time, [reference] } & $\begin{array}{c}\text { Peritoneal } \\
\text { recurrence } \\
\text { alone (\%) }\end{array}$ & Combined (\%) \\
\hline Korea, 1987-1995 [16] & $33.9 \%$ & $43.9 \%$ \\
USA, 1985-2000 [18] & 13.6 & 29.5 \\
Munich, Germany, 2000-2005 [19] & 24 & 34 \\
Italy,1998-2009 [17] & n.r & 30.3 \\
Heildelberg, Germany, n.r. [20] & 10.2 & 39.8 \\
n.r. = not reported & & \\
\hline
\end{tabular}

\section{Materials and methods}

A dual search of pubmed database was made on 30 November 2012, using the following keywords 'gastric' and 'cancer' and 'HIPEC', and 'gastric' and 'cancer' and 'catumaxomab', respectively for the first and the second research. In both cases the filters activated were 'clinical trial' and 'review'. Relatively to the first search were obtained 17 scientific articles, while for the second 9. A subsequent manual search identified additional scientific papers on which this update was made.

\section{Prevention and treatment of peritoneal carcinomato- sis from gastric cancer}

The prognosis of peritoneal carcinomatosis from gastric cancer

Patients with PCGC show a particularly poor prognosis with a five-year survival less than $3 \%$ [23] and an overall mean and median survival of 6.5 months (range 0.1-48.0 months) and 3.1 months, respectively [24]. Among the nongynecologic malignancies the PCGC has a better prognosis than peritoneal carcinomatosis from pancreatic cancer (overall mean and median survival times 2.9 months, range 0.3-13.6 months and 2.1 months respectively), but worse than peritoneal carcinomatosis from colorectal carcinoma (overall mean and median survival times 6.9 months, range 0.6-44.9 months and 5.2 months, respectively) [24]. Saito and collaborators reported that the 5-year survival rate of AGC with FPTCs was $15.3 \%$, almost the same $(14.8 \%)$ as that with disseminating metastasis to the adjacent peritoneum of the stomach. Instead there were no 5 years survivors among patients with metastases to the distant peritoneum [25]. Moreover, a study by Liu and collaborators showed that the 5 years survival rate of patients with microscopic PCGC (i.e. when some tumor nodules are occasionally found in the peritoneum of GC patients by hysto-pathological examination) was $24 \%$ [26].

\section{Results}

Systemic chemotherapy for advanced gastric cancer or peritoneal carcinomatosis from gastric cancer

It has been established that systemic chemotherapy improves median survival in advanced and/or metastatic GC to not more than 12 months [27-30]. However the same gain in term of survival has not been described in patients with macroscopic PCGC [31-34], seemingly because diffusion of systemically administered drugs into the abdominal cavity is inadequate, as if the peritoneal cavity is considered a 'pharmacological sanctuary' [35]. Yonemura and collaborators have obtained better results in patients with PFTCs after radical resection using adjuvant systemic chemotherapy. The patients treated with systemic adjuvant chemotherapy survived significantly longer than that of control group: the 1-year survival rates of control group and adjuvant group were $44 \%$ and $88 \%$, respectively, and the 2 year survival rates of control group and adjuvant group were $9 \%$ and $53 \%$, respectively. The mean overall survival was 21.1 months and 9.1 months for adjuvant group and for control group respectively $(p<0.05)$ [36]. It is assumed that the ineffectiveness of systemic chemotherapy for PCGC is due to the presence of a blood-peritoneal barrier, poor blood supply and oxygenation of cancer cells in the peritoneum and low apoptotic potential of such hypoxic tumor cells [28-39]. In fact, unlike the systemic administration of chemotherapy, the intraperitoneal delivery of chemotherapeutic drugs achieves high regional concentrations while retaining low systemic drug levels. This pharmacokinetic gain is due to the existence of a blood-peritoneal barrier, which keeps a constant high concentration gradient of cytotoxic drug between the peritoneal cavity and the plasma compartment $[37,40]$. Although the precise anatomical nature of blood-peritoneal barrier has not been completely clarified, it has been shown that the broad removal of the diseased peritoneum during cytoreductive surgery (CRS) doesn't look to affect the pharmacokinetics of intraperitoneal chemotherapy [41].

Systemic neoadjuvant chemotherapy for peritoneal carcinomatosis from gastric cancer

Systemic neoadjuvant chemotherapy (NACT) has been described to decrease the load of macroscopic PCGC $[39,42]$. Yano et al. [43] reported a complete remission of peritoneal metastasis in 4 over 26 patients (15.4\%) with PCGC following NACT, all of whom subsequently underwent curative resection. Inokuchi et al. [44] showed partial response in 9 of 13 patients (69\%) following NACT for PCGC. Unexpectedly (but only for those who don't take in account the presence of a blood-peritoneal barrier) it seems that NACT does not greatly influence the elimination (but even the development) of FPTCs. A German study [45] has shown that changes from FPTC positivity to negativity and vice versa can be detected after NACT, irrespective of response to the systemic treatment. In this study, 10 among 42 (24\%) of the patients changed 
to FPTC positivity during NACT, whereas 7 among 19 (37\%) with FPTC positive cytology at staging laparoscopy turned negative.

Hyperthermic intraperitoneal chemotherapy for advanced gastric cancer or peritoneal carcinomatosis from gastric cancer

With these premises it was commonly recognized that in GC peritoneal spread remains a major problem in the management of this neoplasm and, considering the failure of surgery at the peritoneum, there was no role for surgery once the diagnosis of PCGC has been made [46]. To increase effectiveness of intra abdominal treatments, since the eighties the Japanese surgeons combined CRS, regional hyperthermia, and intraperitoneal chemotherapy in a multimodal approach [47]. As for other types of PC, even in case of GC, hyperthermic intraperitoneal chemotherapy (HIPEC) after CRS is accomplished to eliminate FPTCs and to inhibit or delay PC $[46,48]$. CRS must be as complete as possible in order to obtain the best results. The extent of CRS in case of PC has been determined by the Sugar baker's completeness of cytoreduction (CC) score. In this score the CC-0 indicates no residual peritoneal disease after CRS; CC-1, less than $2.5 \mathrm{~mm}$ of residual disease; CC-2, residual tumor between $2.5 \mathrm{~mm}$ and $2.5 \mathrm{~cm}$; and CC-3, more than $2.5 \mathrm{~cm}$ of residual tumor or the presence of a sheet of unresectable tumor nodules [49].

On one hand, some RCTs showed since the early nineties a significant reduction in the rate of subsequent PC and an increase in survival of patients with AGC when radical surgery was combined with HIPEC in an adjuvant manner [8, 50-55], and Yonemura and collaborators demonstrated that in patients with PFTCs HIPEC could improve significantly the median survival time from 15 to 48 months and the 5 years survival rate from $12 \%$ to $42 \%$ [56]. Yan and collaborators in a meta-analysis indicated that HIPEC with or without early postoperative intraperitoneal chemotherapy (EPIC) after primary resection of AGC is associated with improved overall survival. They reported a significant improvement in survival associated with HIPEC alone (hazard ratio = $0.60,95 \%$ confidence interval $(\mathrm{CI})=0.43-40.83, \mathrm{p}=0.002$ ) or this regimen combined with EPIC (hazard ratio $=0.45$, $95 \%$ confidence interval $=0.29-20.68, p=0.0002$ ) [52] .

Nevertheless on the other hand, the treatment of PCGC with CRS and HIPEC seemed to be among the entire PC that with less encouraging results both in terms of survival and of morbidity and mortality $[57,58]$. The French authors in a retrospective, multicenter cohort study published in 2010 [59], evaluated toxicity and principal prognostic factors after CRS and HIPEC (and/or EPIC) for PC from non-gynecologic neoplasms. The study involved 1290 patients from 25 French institutions who underwent 1344 CRS between February 1989 and December 2007, where HIPEC was made in 1154 cases (86.4\%). Although the principal origin of PC was colorectal cancer $(n=523$, $40.5 \%)$, the GC was the third more represented ( $n=159$,
$12.3 \%$ ). With a median follow-up of 45.3 months the whole group of patients included in the study showed an overall 3 and 5 years survival rates of $49 \%$ and $37 \%$, respectively, but the patients with PCGC displayed the worse outcome with an overall 3 and 5 years survival rates of $18 \%$ and $13 \%$, respectively. The overall median survival of the whole group of patients included in the study was 34 months, but was only 9 months for patients with PCGC.

Then still in $2010 \mathrm{Li}$ et al. [60] from China reported the results of their study, where in a group of 128 patients with PCGC, 54 of them (42.2\%) underwent gastrectomy, of which 10 patients underwent resection with HIPEC and the other 74 (57.8\%) underwent non-resection surgery. The median survival in the unresected group was 6.0 months compared to 11.8 months of in the resected patients and they observed a significantly improved survival in the patients that were treated with CRS and HIPEC compared to those that were treated with CRS alone. Although no patient died from resection-related causes, the incidence of overall postoperative complications was higher for the resection with HIPEC group than for the resection alone group ( $20 \%$ vs. $13.2 \%$, however, the difference was not significant, $\mathrm{p}=0.34$ ). This report revealed once again the safety and the efficacy of CRS and HIPEC in PCGC, but despite this evidence supporting radical surgery and HIPEC over surgery alone or palliative chemotherapy, only a minority of all patients in this cohort was treated with HIPEC.

The next year, in 2011, Gill and collaborators published a systematic review of survival, mortality and morbidity regarding the treatment of PCGC by CRS and HIPEC [61]. They selected for inclusion in this review studies published from 2000 to 2010 with non-randomized controlled trials, randomized controlled trials, prospective cohort series and retrospective case series ( $>5$ cases), including adult ( $>18$ years old) patients with PCGC (without other sites of metastatic disease, e.g., liver, lung) who underwent CRS (peritonectomy) combined with HIPEC (the primary gastric resection may be completed at the same surgery as the CRS or at a separate procedure, respectively synchronous or metachronous PCGC). A total of 10 primary studies meeting the inclusion criteria was identified and analysed, including 1 non-randomized prospective controlled trial, 6 prospective case series and 3 retrospective case series with a total of 441 patients with an average age of 48.5 years (range $48-55$ years) and a median follow-up of 46 months (range 19-74 months). In the included studies either open or closed HIPEC technique was utilized, the most common chemotherapeutic agents were cisplatin and mitomycine, with intra abdominal temperatures typically between 40 and $44^{\circ} \mathrm{C}$ and duration between 30 and $120 \mathrm{~min}$. The authors reported an overall median survival of 7.9 months (range: 6.1-9.2 months) (15 months, range: 9.5-43.4 months, for patients with the completeness of cytoreduction (CC) scores of 0 or 1 , i.e. residual nodules after CRS with size less than 2.5 
$\mathrm{mm}$ ) with a 1-year survival of $43 \%$ (range: $22-68 \%$ ) and a 5-year survival of $13 \%$. The treatment-related overall mortality rate was $4.8 \%$ and the overall morbidity was $21.5 \%$ with abscess, fistula and anastomotic leak being the most common complications reported. The length of hospital stay ranged between 7 and 16 days with an ICU stay ranging between 1 and 3 days. Although without any level I evidence, it could be concluded that in PCGC CRS with HIPEC may improve survival with acceptable morbimortality.

The evidence lacking in this review became available in the same year, 2011, when Yang and collaborators published the final results of a phase III RCT, performed in China in order to evaluate the efficacy and safety of CRS plus HIPEC for the treatment of PCGC [62]. The authors included adult (age 20-75 years old) patients with either synchronous or metachronous PCGC, without any lung and liver metastasis or prominent retroperitoneal lymph node metastasis, who were randomized into two arms where the only variable in study after the CRS was the use of HIPEC (open technique, with $120 \mathrm{mg}$ of cisplatin and $30 \mathrm{mg}$ of mitomycin $\mathrm{C}$ each dissolved in $6 \mathrm{l}$ of saline infused into the peritoneal cavity at a rate of $500 \mathrm{ml} / \mathrm{min}$ and a temperature of $43.0 \pm 0.5^{\circ} \mathrm{C}$ for $60-90 \mathrm{~min}$ ). Sixtyeight PCGC patients, including 35 men and 33 women, aged 24-75 years (median 50 years) were randomized into CRS alone $(\mathrm{n}=34)$ and CRS and HIPEC $(\mathrm{n}=34)$ groups with a good balancing regarding major baseline clinicopathological characteristics and surgical procedures. After a median follow-up of 32 months (7.5-83.5 months) the median overall survival was 6.5 months (95\% CI 4.88.2 months) in CRS alone group and 11.0 months (95\% CI 10.0-11.9 months) in the CRS+HIPEC group ( $\mathrm{p}=0.046)$. This outcome was even more significant in patients with synchronous PCGC $(\mathrm{n}=51)$, where the median overall survival was 12.0 months ( $95 \%$ CI 8.1-15.9 months) in CRS+HIPEC group $(\mathrm{n}=24)$ and 6.5 months $(95 \%$ CI $5.0-$ 8.0 months) in the CRS alone group $(\mathrm{n}=27)(\mathrm{p}=0.029)$. The 1, 2 and 3 year survival rates were 29.4, 5.9 and $0 \%$ for CRS group and $41.2,14.7$ and $5.9 \%$ for CRS+HIPEC group. The CC influenced the survival, but HIPEC obtained a significant advantage either in CC $0-1$, either in CC 2-3 patients. In the CRS+HIPEC patients, the median overall survival was 12.0 months (95\% CI 8.1-16.0 months) and 8.2 months (95\% CI $0.5-16.5$ months) in CC $0-1(n=20)$ and in CC $2-3$ subgroup $(\mathrm{n}=14)$ respectively, $(\mathrm{p}=0.000)$. In CRS alone patients, the median overall survival was 11.0 months ( $95 \% \mathrm{CI} 8.8-13.2$ months) and 4.0 months (95\% CI 1.3-6.8 months) in CC 0-1 $(\mathrm{n}=20)$ and in CC $2-3$ subgroup $(\mathrm{n}=14)$ respectively, $(\mathrm{p}=0.000)$. Serious adverse events (SAE), including wound infection and sepsis, respiratory failure, gastrointestinal bleeding, severe bone marrow suppression and intestinal obstruction, arose in 9 patients, 4 in the CRS alone group (11.7\%) and 5 in the CRS+HIPEC group (14.7\%) $(\mathrm{p}=0.839)$. Multivariate analysis recognized CRS+HIPEC, synchronous PC, CC 0-1, systemic chemotherapy and no SAE as major independent predictors for better survival. HIPEC was about 2.6 times more likely to increase survival (hazard ratio $=2.617$, $95 \%$ confidence interval $=1.436-4.769)$. From the methodological point of view, it has to be pointed out that this is the first RCT in patients with established PC where the only variable in the study was HIPEC (unlike the Dutch study [63] regarding the PC from colorectal cancer), showing an advantage in term of survival. It has to be concluded that HIPEC after CRS improves survival with acceptable morbidity in patients with PCGC especially when synchronous. These advances in the management for PCGC encourage the use of any diagnostic means, including staging laparoscopy, in order to plan carefully HIPEC after CRS and other multimodal treatments [64].

\section{A new drug for intraperitoneal treatment of peritoneal carcinomatosis from gastric cancer: Catumaxomab}

In the last ten years an interesting new drug to be applied for intraperitoneal treatment of GC has been developed in Germany. Catumaxomab [65] (trade name Removab ${ }^{\circledR}$ ) is a rat-mouse hybrid monoclonal antibody that is made up of one 'half' (one heavy chain and one light chain) of an anti-epithelial cell adhesion molecule (EpCAM) antibody and one half of an anti-CD3 antibody, binding both EpCAM and CD3. EpCAM is an epithelial differentiation antigen that is expressed in normal epithelial cells and on almost all carcinomas (especially gastrointestinal and ovarian carcinomas) and functions as a cell adhesion molecule. In addition, the Fc-region can bind to an Fc receptor on accessory cells like other antibodies, which has led to calling the drug a trifunctional antibody. Actually catumaxomab is used to treat malignant ascites, because the intraperitoneal application of this anti-EpCAM antibody has shown significant benefits in puncture-free survival (survival without repeated paracentesis) for patients with malignant ascites in a phase II/III randomized trial [66]. In this study although the difference in median OS (secondary endpoint) for the whole group of patients (72 days for paracentesis plus catumaxomab compared to 68 days for paracentesis alone, $\mathrm{p}=0.08$ ) was not significant, the same outcome (i.e. the difference in OS) was statistically significant in patients with GC (median 71 vs 44 days; $p=0.03$ ) [67]. The same result, i.e. an improved progression-free survival, has been replicated in phase II studies [67] with the use of intraperitoneal catumaxomab in gastrointestinal EpCAM+ tumors. Furthermore two phase 2 studies are ongoing (follow-up phase) where resectable AGC patients are treated with adjuvant catumaxomab. The first study [68] included 55 patients randomized to surgery plus catumaxomab $(10 \mu \mathrm{g}$ catumaxomab infused directly after surgery intra-operative, followed by four ascending intraperitoneal doses) or surgery alone. A total of $78 \%$ of the patients received all five catumaxomab infusions and there were no clinically relevant differences in the incidence of surgical complications between the surgery alone and the surgery plus catumaxomab group. In the second study [69], 54 patients with radically resected AGC were treated intra-operatively and i.p. in adjuvant setting with catumaxomab after they received NACT. For both studies final results are awaiting. 


\section{Conclusions}

PCGC is a common conditions and is still considered to be a terminal event of systemic metastasis by the vast majority of the scientific community. Nonetheless the present review shows that the PCGC can be prevented and treated by the use of multimodal treatments incorporating CRS with either systemic and locoregional intraperitoneal chemotherapy (both neoadjuvant and adjuvant). In case of AGC and FPTCs positive patients HIPEC, as adjuvant treatment, has shown the ability to significantly prevent the establishment of PCGC. In PCGC, CRS and HIPEC have shown to improve survival with acceptable morbimortality. So it is very important to obtain the diagnosis of PCGC before the CRS and HIPEC with the use of staging laparoscopy. However, it was clarified that in patients with PCGC, multimodal treatment should be mandatory used, leaving a pivotal role to HIPEC after CRS. Yonemura and collaborators proposed the following strategy: a bidirectional chemotherapy called neoadjuvant intraperitoneal/systemic chemotherapy, followed by CRS and HIPEC and eventually completed by an EPIC [70]. A different approach can be NACT followed by CRS and HIPEC with the associated use either preoperatively or postoperatively of intraperitoneal catumaxomab.

In the future the prevention and treatment of PCGC will be increasingly characterized by the need to combine several therapies (surgical and non-surgical), either simultaneously or sequentially.

\section{Conflict of interest}

All authors declare no conflict of interest

\section{References}

[1] Bertuccio P, Chatenoud L, Levi F, Praud D, Ferlay J, et al. (2009) Recent patterns in gastric cancer: a global overview. Int J Cancer 125:666-673.

[2] Ferlay J, Shin HR, Bray F, Forman D, Mathers C, et al (2010) Estimates of worldwide burden of cancer in 2008: GLOBOCAN 2008. Int J Cancer 127:2893-28917.

[3] Nakamura K, Ueyama T, Yao T, Xuan ZX, Ambe K, et al. (1992) Pathology and prognosis of gastric carcinoma. Findings in 10,000 patients who underwent primary gastrectomy. Cancer 70:10301037.

[4] Takahashi T, Hagiwara A, Sawai K, Kondo S, Yamaguchi T (1991) Intensive Intraoperative Local Chemotherapy for Lymph Node and Peritoneal Metastases in Gastric Cancer. Onkologie 14:152-157.

[5] Yu CC, Levison DA, Dunn JA, Ward LC, Demonakou M, et al. (1995) Pathological prognostic factors in the second British Stomach Cancer Group trial of adjuvant therapy in resectable gastric cancer. Br J Cancer 71:1106-1110.

[6] Ikeguchi M, Oka A, Tsujitani S, Maeta M, Kaibara N (1994) Relationship between area of serosal invasion and intraperitoneal free cancer cells in patients with gastric cancer. Anticancer Res 14:2131-2134.

[7] Gunderson LL, Sosin H (1982) Adenocarcinoma of the stomach: areas of failure in a re-operation series (second or symptomatic look) clinicopathologic correlation and implications for adjuvant therapy. Int J Radiat Oncol Biol Phys 8:1-11.

[8] Hamazoe R, Maeta M, Kaibara N (1994) Intraperitoneal thermochemotherapy for prevention of peritoneal recurrence of gastric cancer. Final results of a randomized controlled study. Cancer 73:2048-2052.
[9] Ikeguchi M, Kondou A, Oka A, Tsujitani S, Maeta M, et al. (1995) Effects of continuous hyperthermic peritoneal perfusion on prognosis of gastric cancer with serosal invasion. Eur J Surg 161:581-586.

[10] Koga S, Hamazoe R, Maeta M, Shimizu N, Murakami A, et al. (1988) Prophylactic therapy for peritoneal recurrence of gastric cancer by continuous hyperthermic peritoneal perfusion with mitomycin C. Cancer 61:232-237.

[11] Kuramoto M, Shimada S, Ikeshima S, Matsuo A, Yagi Y, et al. (2009) Extensive intraoperative peritoneal lavage as a standard prophylactic strategy for peritoneal recurrence in patients with gastric carcinoma. Ann Surg 250:242-246.

[12] Bando E, Yonemura Y, Takeshita Y, Taniguchi K, Yasui T, et al. (1999) Intraoperative lavage for cytological examination in 1,297 patients with gastric carcinoma. Am J Surg 178:256-262.

[13] Wu CC, Chen JT, Chang MC, Ho WL, Chen CY, et al. (1997) Optimal surgical strategy for potentially curable serosa-involved gastric carcinoma with intraperitoneal free cancer cells. J Am Coll Surg 184:611-617.

[14] Benevolo M, Mottolese M, Cosimelli M, Tedesco M, Giannarelli D, et al. (1998) Diagnostic and prognostic value of peritoneal immunocytology in gastric cancer. J Clin Oncol 16:3406-3411.

[15] Juhl H, Stritzel M, Wroblewski A, Henne-Bruns D, Kremer B, et al. (1994) Immunocytological detection of micrometastatic cells: comparative evaluation of findings in the peritoneal cavity and the bone marrow of gastric, colorectal and pancreatic cancer patients. Int J Cancer 57:330-335.

[16] Yoo CH, Noh SH, Shin DW, Choi SH, Min JS (2000) Recurrence following curative resection for gastric carcinoma. Br J Surg 87:236-242.

[17] Baiocchi L et al. personal communication.

[18] D’Angelica M, Gonen M, Brennan MF, Turnbull AD, Bains M, et al. (2004) Patterns of initial recurrence in completely resected gastric adenocarcinoma. Ann Surg 240:808-816.

[19] Siewert JR, Lordick F, Ott K, Brücher BL, Rosenberg R (2006) Curative vs palliative strategies in locoregional recurrence of gastrointestinal malignancies. Chirurg 77:227-235.

[20] Ott K, Lordick F, Blank S, Büchler M (2011) Gastric cancer: surgery in 2011. Langenbecks Arch Surg 396:743-758.

[21] Yonemura Y, Kawamura T, Bandou E, Tsukiyama G, Endou Y, et al. (2007) The natural history of free cancer cells in the peritoneal cavity. Recent Results Cancer Res 169:11-23.

[22] Wu B, Wu D, Wang M, Wang G (2008) Recurrence in patients following curative resection of early gastric carcinoma. J Surg Oncol 98:411-414.

[23] Yonemura Y (1996) Contemporary approaches towards cure of gastric cancer. Kanazawa, Japan: Maeda Shoten Co115.

[24] Sadeghi B, Arvieux C, Glehen O, Beaujard AC, Rivoire M, et al. (2000) Peritoneal carcinomatosis from non-gynecologic malignancies: results of the EVOCAPE 1 multicentric prospective study. Cancer 88:358-363.

[25] Saito H, Kihara K, Kuroda H, Matsunaga T, Tatebe S, et al. (2011) Surgical outcomes for gastric cancer patients with intraperitoneal free cancer cell, but no macroscopic peritoneal metastasis. J Surg Oncol. 104:534-537.

[26] Liu X, Cai H, Sheng W, Wang Y (2012) Long-term results and prognostic factors of gastric cancer patients with microscopic peritoneal carcinomatosis. PLoS One 7:e37284.

[27] Wagner AD, Unverzagt S, Grothe W, Kleber G, Grothey A, et al. (2010) Chemotherapy for advanced gastric cancer. Cochrane Database Syst Rev 17 :CD004064.

[28] Van Cutsem E, Moiseyenko VM, Tjulandin S, Majlis A, Constenla $\mathrm{M}$, et al. (2006) Phase III study of docetaxel and cisplatin plus fluorouracil compared with cisplatin and fluorouracil as first-line therapy for advanced gastric cancer: a report of the V325 Study Group. J Clin Oncol 24:4991-4997.

[29] Cunningham D, Starling N, Rao S, Iveson T, Nicolson M (2008) Upper Gastrointestinal Clinical Studies Group of the National Cancer Research Institute of the United Kingdom. Capecitabine and oxaliplatin for advanced esophagogastric cancer. N Engl J Med 358:36-46.

[30] Roth AD, Fazio N, Stupp R, Falk S, Bernhard J, et al. (2007) Docetaxel, cisplatin, and fluorouracil; docetaxel and cisplatin; and epirubicin, cisplatin, and fluorouracil as systemic treatment for advanced gastric carcinoma: a randomized phase II trial of the Swiss Group for Clinical Cancer Research.. J Clin Oncol 25:3217-3223. 
[31] Ross P, Nicolson M, Cunningham D, Valle J, Seymour M, et al. (2002) Prospective randomized trial comparing mitomycin, cisplatin, and protracted venous-infusion fluorouracil (PVI 5-FU) With epirubicin, cisplatin, and PVI 5-FU in advanced esophagogastric cancer. J Clin Oncol 20:1996-2004.

[32] Baba H, Yamamoto M, Endo K, Ikeda Y, Toh Y, et al. (2003) Clinical efficacy of S-1 combined with cisplatin for advanced gastric cancer. Gastric Cancer 6:45-49.

[33] Yabusaki H, Nashimoto A, Tanaka O (2003) Evaluation of TS-1 combined with cisplatin for neoadjuvant chemotherapy in patients with advanced gastric cancer. Gan To Kagaku Ryoho 30:19331940.

[34] Preusser P, Wilke H, Achterrath W, Fink U, Lenaz L, et al. (1989) Phase II study with the combination etoposide, doxorubicin, and cisplatin in advanced measurable gastric cancer. J Clin Oncol 7:1310-1317.

[35] Al-Shammaa HA, Li Y, Yonemura Y (2008) Current status and future strategies of cytoreductive surgery plus intraperitoneal hyperthermic chemotherapy for peritoneal carcinomatosis. World J Gastroenterol 14:1159-1166.

[36] Yonemura Y, Endou Y, Bando E, Kawamura T, Tsukiyama G, et al. (2006) The usefulness of oral TS- 1 treatment for potentially curable gastric cancer patients with intraperitoneal free cancer cells. Cancer Therapy 4:135-142.

[37] Jacquet P, Sugarbaker PH (1996) Peritoneal-plasma barrier. Cancer Treat Res 82:53-63.

[38] Goldie JH (1987) Scientific basis for adjuvant and primary (neoadjuvant) chemotherapy. Semin Oncol. 14:1-7.

[39] Graeber TG, Osmanian C, Jacks T, Housman DE, Koch CJ, et al. (1996) Hypoxia-mediated selection of cells with diminished apoptotic potential in solid tumours. Nature 379:88-91.

[40] Flessner MF (2005) The transport barrier in intraperitoneal therapy. Am J Physiol Renal Physiol 288:F433-F442.

[41] de Lima Vazquez V, Stuart OA, Mohamed F, Sugarbaker PH (2003) Extent of parietal peritonectomy does not change intraperitoneal chemotherapy pharmacokinetics. Cancer Chemother Pharmacol 52:108-112.

[42] Kochi M, Fujii M, Kanamori N, Kaiga T, Takahashi T, et al. (2006) Neoadjuvant chemotherapy with S-1 and CDDP in advanced gastric cancer. J Cancer Res Clin Oncol 132:781-785.

[43] Yano M, Shiozaki H, Inoue M, Tamura S, Doki Y, et al. (2002) Neoadjuvant chemotherapy followed by salvage surgery: effect on survival of patients with primary noncurative gastric cancer. World J Surg 26:1155-1159.

[44] Inokuchi M, Yamashita T, Yamada H, Kojima K, Ichikawa W, et al. (2006) Phase I/II study of S-1 combined with irinotecan for metastatic advanced gastric cancer. Br J Cancer 94:1130-1135.

[45] Lorenzen S, Panzram B, Rosenberg R, Nekarda H, Becker K, et al. (2010) Prognostic significance of free peritoneal tumor cells in the peritoneal cavity before and after neoadjuvant chemotherapy in patients with gastric carcinoma undergoing potentially curative resection. Ann Surg Oncol 17:2733-2739.

[46] Averbach AM, Jacquet P (1996) Strategies to decrease the incidence of intra-abdominal recurrence in resectable gastric cancer. Br J Surg 83:726-733.

[47] Yonemura Y, Fujimura T, Fushida S, Takegawa S, Kamata T, et al. (1991) Hyperthermo-chemotherapy combined with cytoreductive surgery for the treatment of gastric cancer with peritoneal dissemination. World J Surg 15:530-535.

[48] Elias Elias D, Detroz B, Debaene B, Damia E, Leclercq B, et al. (1991). Treatment of peritoneal carcinomatosis by intraperitoneal chemo-hyperthermia: reliable and unreliable concepts. Hepatogastroenterology 41:207-213.

[49] Sugarbaker PH (2001) Cytoreductive surgery and peri-operative intraperitoneal chemotherapy as a curative approach to pseudomyxoma peritonei syndrome Eur J Surg Oncol 27:239-243.

[50] Fujimura T, Yonemura Y, Muraoka K, Takamura H, Hirono Y, et al. (1994) Continuous hyperthermic peritoneal perfusion for the prevention of peritoneal recurrence of gastric cancer: randomized controlled study. World J Surg 18:150-155.

[51] Yonemura Y, Ninomiya I, Kaji M, Sugiyama K, Fujimura K, et al. (1995) Prophylaxis with intraoperative chemohyperthermia against peritoneal recurrence of serosal invasion-positive gastric cancer. World J Surg 19:450-454.
[52] Yan TD, Black D, Sugarbaker PH, Zhu J, Yonemura Y, et al. (2007) A systematic review and meta-analysis of the randomized controlled trials on adjuvant intraperitoneal chemotherapy for resectable gastric cancer. Ann Surg Oncol 14:2702-2713.

[53] Xu DZ, Zhan YQ Sun XW, Cao SM, Geng QR (2004) Meta-analysis of intraperitoneal chemotherapy for gastric cancer. World J Gastroenterol 10:2727-2730.

[54] Fujimoto S, Takahashi M, Mutou T, Kobayashi K, Toyosawa T (1999) Successful intraperitoneal hyperthermic chemoperfusion for the prevention of postoperative peritoneal recurrence in patients with advanced gastric carcinoma. Cancer 85:529-534.

[55] Yonemura Y, de Aretxabala X, Fujimura T, Fushida S, Katayama K, et al. (2001) Intraoperative chemohyperthermic peritoneal perfusion as an adjuvant to gastric cancer: final results of a randomized controlled study. Hepatogastroenterology 48:1776-1782.

[56] Yonemura Y, Bando E, Kawamura T, Ito H, Endo Y, et al. (2007) Cytoreductionand intraperitoneal chemotherapy for carcinomatosis from gastric cancer. Cancer Treat Res 134:357-373.

[57] Hall JJ, Loggie BW, Shen P, Beamer S, Douglas Case L, et al. (2004) Cytoreductive surgery with intraperitoneal hyperthermic chemotherapy for advanced gastric cancer. J Gastrointest Surg 8:454-463.

[58] Samel S, Singal A, Becker H, Post S (2000) Problems with intraoperative hyperthermic peritoneal chemotherapy for advanced gastric cancer. Eur J Surg Oncol 26:222-226.

[59] Glehen O, Gilly FN, Boutitie F, Bereder JM, Quenet F, et al. (2010) Toward curative treatment of peritoneal carcinomatosis from nonovarian origin by cytoreductive surgery combined with perioperative intraperitoneal chemotherapy: a multi-institutional study of 1,290 patients. Cancer 116:5608-5618.

[60] Li C, Yan M, Chen J, Xiang M, Zhu ZG, et al. (2010) Surgical resection with hyperthermic intraperitoneal chemotherapy for gastric cancer patients with peritoneal dissemination. J Surg Oncol 102:361-365.

[61] Gill RS, Al-Adra DP, Nagendran J, Campbell S, Shi X, et al. (2011) Treatment of gastric cancer with peritoneal carcinomatosis by cytoreductive surgery and HIPEC: a systematic review of survival, mortality, and morbidity J Surg Oncol 104:692-698.

[62] Yang XJ, Huang CQ Suo T, Mei LJ, Yang GL, et al. (2011) Cytoreductive surgery and hyperthermic intraperitoneal chemotherapy improves survival of patients with peritoneal carcinomatosis from gastric cancer: final results of a phase III randomized clinical trial. Ann Surg Oncol 18:1575-1581.

[63] Verwaal VJ, Bruin S, Boot H, van Slooten G, van Tinteren H (2008) 8-year follow-up of randomized trial: cytoreduction and hyperthermic intraperitoneal chemotherapy versus systemic chemotherapy in patients with peritoneal carcinomatosis of colorectal cancer. Ann Surg Oncol 15:2426-2432.

[64] Muntean V, Mihailov A, Iancu C, Toganel R, Fabian O, et al. (2009) Staging laparoscopy in gastric cancer. Accuracy and impact on therapy. J Gastrointestin Liver Dis 18:189-195.

[65] Linke R, Klein A, Seimetz D (2010) Catumaxomab: clinical development and future directions. MAbs 2:129-136.

[66] Heiss MM, Murawa P, Koralewski P, Kutarska E, Kolesnik O0, et al. (2009) The trifunctional antibody catumaxomab for the treatment of malignant ascites due to epithelial cancer: Results of a prospective randomized phase II/III trial. Int J Cancer 127:2209-2221.

[67] Ströhlein MA, Lordick F, Rüttinger D, Grützner KU, Schemanski OC, Immunotherapy of peritoneal carcinomatosis with the antibody catumaxomab in colon, gastric, or pancreatic cancer: an open-label, multicenter, phase I/II trial. Onkologie 34:101-108.

[68] Schuhmacher CP, Ridwelski K, Atanackovic D, Arnold D, Wöll Ewald, et al. (2011) One-year follow-up data for Catumaxomab as part of a multimodal approach in patients with primarily resectable gastric cancer. Ann Onc 22 (Suppl.5), v11.

[69] Krueger CM, Berdov B, Roman L, Luft A, Lampe P, et al. (2008) Intraoperative, adjuvant treatment of gastric cancer with the trifunctional antibody catumaxomab compared to surgery alone: a phase II study. Ann Onc 19 (Suppl 8) viii172.

[70] Yonemura Y, Endou Y, Sasaki T, Hirano M, Mizumoto A, et al. (2010) Surgical treatment for peritoneal carcinomatosis from gastric cancer. Eur J Surg Oncol 36:1131-1138. 